\title{
Rastreamento Populacional de Anormalidades \\ Cardíacas Fetais por Ecocardiografia Pré-Natal \\ em Gestações de Baixo Risco no Município de Porto Alegre
}

\author{
Lauro L. Hagemann, Paulo Zielinsky \\ Porto Alegre, RS
}

\section{Objetivo}

Estudar as anormalidades morfo-funcionais do sistema cardiovascular fetal detectáveis por ultra-sonografia.

\section{Métodos}

Foram submetidos 3.980 fetos de gestantes sem risco obstétrico ou cardiológico, do município de Porto Alegre, de julho/ 1996 a novembro/2000, ao ecocardiograma de rastreamento para malformações cardiovasculares através dos cortes de quatro câmaras e vias de saída dos ventrículos direito e esquerdo.

\section{Resultados}

Houve 103 diagnósticos de anormalidades cardiovasculares fetais, correspondendo a 2,5\% (103/3.980) da população estudada, ou 25,8/1000, sendo que 47 referiam-se a alterações morfofuncionais do coração fetal, prevalência global de 11,8/1000 (47/3.980), e 56 exibiam alterações na refringência (golf ball). Três falso-negativos e nenhum falso-positivo elevaram a prevalência global para 12.5/1000(50/3.980), ou 26,6/1000(106/3.980), quando incluídas também as alterações de refringência.

\section{Conclusão}

A detecção das malformações cardiovasculares ainda no período intra-uterino, visando planejamento perinatal é possível, confirmando a experiência internacional. É sugerida implantação do rastreamento de malformações cardiovasculares, aninhada em rede de atenção rotineira de ultra-sonografia obstétrica.

\section{Palavra-chave}

anormalidades cardíacas fetais; ecocardiografia pré-natal; gestações; mortalidade neonatal

Instituto de Cardiologia do Rio Grande do Sul/Fundação Universitária de Cardiologia

Endereço para Correspondência: Unidade de Pesquisa do IC/FUC - Dr. Lauro Luís Hagemann - Av. Princesa Isabel, 370 - Cep 90620-001 Porto Alegre.RS. E-mail: pesquisa@cardnet.tche.br

Recebido para Publicação em 28/3/01

Aceito em 23/6/03
As cardiopatias congênitas estão entre as malformações mais comuns em fetos humanos e, como grupo, consideradas as mais freqüentes ${ }^{1}$. Pelo seu mau prognóstico, contribuem significativamente para a mortalidade infantil, tornando-se responsáveis por cerca de $10 \%$ dos óbitos infantis e metade das mortes por malformação congênita².

Os coeficientes de mortalidade neonatal, em 1998, foram, respectivamente para o Rio Grande do Sul e Porto Alegre, de 10,0/1000 e 8,3/1000 nascidos vivos. Em 1999, esses índices voltaram a sofrer queda, determinando uma taxa de 9,6/1000 para o Rio Grande do Sul e de 7,1/1000 nascimentos vivos em Porto Alegre ${ }^{3.5}$, dados ainda não publicados, cedidos pela Secretaria Municipal da Saúde e Serviço Social e pela Secretaria Estadual da Saúde do Rio Grande do Sul.

Os óbitos ocorridos no período neonatal (170/291), especialmente na $1^{\text {a }}$ semana de vida(121/170), seguem sendo responsáveis por mais da metade $(58,4 \%)$ da mortalidade infantil de Porto Alegre, em 1999, e, ao se observar a mortalidade pós-natal, identifica-se que $61,2 \%$ dos óbitos de 28 a 364 dias ocorrem nos três primeiros meses de vida (dados também ainda não publicados, informados pela Secretaria Municipal da Saúde e Serviço Social e pela Secretaria Estadual da Saúde do Rio Grande do Sul).

As principais causas de mortalidade neonatal em Porto Alegre (194 óbitos de 0.27 dias de vida) foram, em 1998, em ordem decrescente de importância, a síndrome do desconforto respiratório ( 25 casos), a prematuridade (24 casos), as malformações cardiovasculares (23 casos) e repercussões fetais de complicações maternas da gestação (18 casos) ${ }^{3 \cdot 5}$, responsáveis por cerca de $50 \%$ do obituário nessa faixa etária. Em 1998, as afecções do período perinatal e as anomalias congênitas, respectivamente 1은 e $2 \circ$ grupo de causas mais importantes de mortalidade infantil, responderam por 39,5\% e 20,7\% da mortalidade infantil em Porto Alegre, perfazendo 60,2\% dos óbitos ocorridos em menores de um ano (227 de um total de 377).

As malformações cardiovasculares constituem-se no principal grupo de causas (Classificação Internacional de Doenças, $10^{a}$ revisão, códigos Q20 a Q25) de óbito infantil por malformações congênitas, sendo responsáveis por cerca de um terço das mortes nesse grupo específico e podendo atingir, como em 1999, quase a metade. Nesse ano, 7,9\% (23/291) do total de óbitos em menores de um ano em Porto Alegre deveu-se a malformações cardiovasculares (dados ainda não publicados, informados pela Secreta- 
ria Municipal da Saúde e Serviço Social e pela Secretaria Estadual da Saúde do Rio Grande do Sul).

Estudos de prevalência global ${ }^{2,6 \cdot 15}$ de cardiopatias congênitas realizados no período pós-natal à disposição na literatura médica mostram uma variação de 3,5 a 13,7/1000 nascidos vivos, e foram realizados em épocas e regiões diferentes e com metodologias não padronizadas, tornando-se temerárias comparações entre eles.

É importante salientar, quando se deseja estudar a prevalência total de cardiopatias congênitas, que a prevalência em natimortos é aproximadamente 10 vezes maior que em recém-nascidos vivos $^{16 \cdot 19}$.

Apesar da pouca informação sobre prevalência de cardiopatias congênitas em abortos disponível na literatura ${ }^{19-21}$, estima-se que, caso se agregasse aos números conhecidos as cardiopatias congênitas presentes em abortos e natimortos, a prevalência global de cardiopatias congênitas seria multiplicada por $5^{22}$.

Embora seja importante selecionar grupos com risco aumentado para malformações cardiovasculares, somente $10 \%$ dos recém. nascidos vivos com cardiopatia congênita têm um fator de risco identificável na gestação ${ }^{23}$.

Vários autores relatam prevalências de cardiopatias congênitas com diagnóstico pré-natal em diferentes países nos últimos 15 anos $^{24 \cdot 33}$. Observa-se uma grande variação nos índices apresentados, de 3,3 ${ }^{24}$ a 14,9/1000 recém-nascidos vivos ${ }^{25}$, atribuindo-se a isso as diferenças de tecnologia e delineamento empregados.

Constituindo uma pequena percentagem de todas as doenças cardíacas, as cardiopatias congênitas, no entanto, são responsáveis por significativa parcela da mortalidade, morbidade, incapacidade e potencial perda de anos de vida com qualidade, afetando especialmente menores de um ano, não sendo conhecido nenhum estudo que tenha se proposto a determinar a prevalência de malformações cardiovasculares nessa população no município de Porto Alegre.

Diante do exposto, objetivou-se determinar a prevalência global e específica das anormalidades morfofuncionais do sistema cardiovascular fetal detectáveis pelos cortes ecocardiográficos de 4 câmaras, vias de saída dos ventrículos direito e esquerdo e arco aórtico em uma população de gestantes de baixo risco, realizando-se estudo transversal (estudo de prevalência) em parte da área geográfica do município de Porto Alegre.

\section{Métodos}

Todas as gestantes em acompanhamento pré-natal em postos de saúde ou unidades sanitárias, locais onde se realiza o pré-natal de baixo risco no município de Porto Alegre, eram elegíveis para o estudo, e foram recrutadas, de modo consecutivo e não intencional, entre julho/1996 a novembro/2000, não tendo sido aceitos fetos de gestantes de outros municípios.

O rastreamento de malformações cardiovasculares fetais foi feito através da visibilização dos cortes de quatro câmaras cardíacas, vias de saída dos ventrículos direito e esquerdo e arco aórti$\mathrm{co}^{23,34,35}$, utilizando-se ecógrafos obstétricos Shimadzu 350, dotado de transdutor convexo de 3,5 $\mathrm{MHz}$, com capacidade para ima. gem bidimensional e modo M, Aloka 500, dotado de transdutor convexo de 3,5 MHz, com capacidade para imagem bidimensional 314 e modo M e Medson Eureka, dotado de transdutor convexo de
3,5 MHz, com capacidade para imagem bidimensional e modo M. Além desses, foram utilizados os equipamentos da Unidade de Cardiologia Fetal do Instituto de Cardiologia/Fundação Universitária de Cardiologia ATL Ultramark-9 Digital Plus, Acuson XP-10 e Acuson Aspen. Não foram realizadas mensurações rotineiras das estruturas identificadas.

Na impossibilidade de deslocar todas as gestantes em estudo, realizaram-se os ecocardiogramas nas unidades de saúde, com aparelho de ultra-sonografia que permitisse a visibilização do coração fetal.

Os ecocardiogramas fetais eram oferecidos como parte da rotina de atenção pré-natal, livres de ônus, após a $18^{a}$ semana de gestação, às gestantes que aderissem espontaneamente aos estudos, após esclarecimentos (aprovação do protocolo número UP 1673/95 pelo Comitê de Ética em Pesquisa do Instituto de Cardiologia).

A equipe de examinadores que visitava os postos de atenção primária constituía-se idealmente de um médico com treinamento na obtenção das imagens de triagem (não necessariamente ecocardiografista), uma secretária, um ou mais acadêmicos (bolsistas de iniciação científica) ou estagiários da Unidade de Cardiologia Fetal, uma psicóloga, uma assistente social ou estagiárias desses setores, que auxiliavam na obtenção dos dados e na execução de projetos aninhados no projeto maior de detecção pré-natal de cardiopatias congênitas.

Gestantes que, preenchendo os requisitos necessários à participação no estudo, espontaneamente tomavam conhecimento do programa de detecção, ou as que, por outros motivos, perdiam a oportunidade de realizar o exame em seu posto de referência, ou estavam em idade gestacional mais adiantada e com data de exame no posto de saúde de referência marcada para após a sua data provável de parto, agendavam data de exame com a equipe de rastreamento nas dependências do Instituto de Cardiologia.

Concomitantemente ao ecocardiograma fetal eram obtidos dados sobre a gestação em curso (dados de identificação, número de gestações e paridade, história médica e obstétrica pregressa, evolução e intercorrências na gestação atual) na busca de fatores que pudessem evidenciar risco para malformações cardiovasculares fetais (diabetes mellitus, colagenose, fenilcetonúria, uso de medicamentos como indometacina, lítio e fenitoína, tabagismo, alcoolismo, consangüinidade, história familiar ou gestações anteriores com cardiopatia congênita, infecções congênitas, arritmia, malformações não cardíacas, retardo de crescimento, hidropisia, oligo/ polidramnio).

Uma vez suspeitada a malformação cardiovascular fetal, a gestante era encaminhada imediatamente para ecocardiograma em nível terciário de atenção na Unidade de Cardiologia Fetal, com agendamento preferencial sobre os demais, com intervalo de tempo não superior a uma semana. Caso confirmada a existência de malformação cardiovascular, o feto era incluído nos protocolos de atenção da Unidade, em geral com a programação de atendimento obstétrico na dependências do Instituto de Cardiologia.

O acompanhamento dos desfechos pós-natais foi realizado obtendo-se informações oriundas das mães, das maternidades e dos serviços de Neonatologia e de Cardiologia Pediátrica onde efetivava-se o parto, e dos arquivos de morbidade e mortalidade fetal e infantil da Secretaria Municipal de Saúde e Serviço Social do município. 
Os dados coletados foram armazenados em banco de dados específico usando o pacote EPIINFO 6.04b (Center for Disease Control (CDC), Atlanta, GA, USA e World Health Organization, Geneve, Switzerland, October 1997).

A análise estatística descritiva (freqüências, médias, medianas, e outras) foi produzida a partir dos dados armazenados nesse banco de dados informatizado.

\section{Resultados}

No período estudado entre julho/1996 e novembro/2000, foram realizados 3.980 ecocardiogramas fetais de triagem em popula. ção de baixo risco obstétrico e cardiológico para malformações cardiovasculares, sendo que 3.263 foram realizados nos postos de atendimento primário e 717 nas dependências da Unidade de Cardiologia Fetal do Instituto de Cardiologia (tab. I).

A idade das pacientes variou de 13 a 48 (média de 25,2) anos, desvio padrão de 6,7 e mediana de 24 anos. O número de gestações oscilou de 1 a 13, com média de 2,5 e desvio padrão de 1,7 e mediana de 2. A paridade variou de 0 a 9, com média de 1 e desvio padrão de 1,4. A escolaridade materna foi de 8 anos (I grau), em média. Predominou o casamento formal entre os ca. sais (39\%) e a renda familiar mediana foi de 3 salários mínimos (salário mínimo $=\mathrm{R} \$ 151,00$ ).

A idade gestacional em que foi praticado o ecocardiograma fetal, informada pela paciente, variou de 14 a 42 semanas com média de 28,6 e desvio padrão de 5,7, sendo que 756 gestantes não sabiam informar com segurança a idade gestacional e tiveram o diâmetro biparietal fetal mensurado durante a realização do exame para estimativa da idade gestacional, que variou de 8 a 41 , com média de 28 e desvio padrão de 6 semanas.

Nessa população, 6,5\% das gestantes relatavam alguma doença sistêmica prévia à gestação (lupus eritematoso sistêmico, diabetes mellitus, hipertensão arterial sistêmica, epilepsia ou cardiopatia congênita), 27,2\% acusavam uso de fármacos e/ou drogas na gestação (fumo, álcool, lítio, indometacina, fenitoína, ácido acetilsalicílico ou anticoncepcional oral) e 3,6\% referiam doença sis. têmica durante a gestação em curso (diabetes melittus, hipertensão arterial sistêmica, pré-eclampsia ou eclampsia). Somente 51 gestantes relataram a presença de cardiopatia congênita em filhos anteriores (tab. II).

A tabela II mostra que 3.263 gestantes (82\%) realizaram ecocardiograma fetal de triagem nos postos de atenção primária, enquanto 717 (18\%) procuraram a Unidade de Cardiologia Fetal para realizar o exame de rastreamento.

\begin{tabular}{|c|c|c|c|}
\hline \multicolumn{4}{|c|}{ Tabela I - População estudada ( $n=3,980)$} \\
\hline & Amplitude & Média ( $\pm \mathrm{DP}$ ) & Mediana \\
\hline Idade materna (anos) & $13-48$ & $25,2 \pm 6,7$ & 24 \\
\hline Gestações & $1 \cdot 13$ & $2,5 \pm 1,7$ & 2 \\
\hline Paridade & 0.9 & $1 \pm 1,4$ & 1 \\
\hline Idade gestacional - DUM (semanas) & 14.42 & $28,6 \pm 5,7$ & 29 \\
\hline Idade gestacional - ECO (semanas) & $8-41$ & $28 \pm 6,0$ & 29 \\
\hline Escolaridade (anos) & $0-15$ & $8,1 \pm 2,9$ & 8 \\
\hline Renda familiar (SM) & 0.99 & $4,9 \pm 5,8$ & 3 \\
\hline
\end{tabular}

Entre os 3.263 pacientes originários dos postos de atendimento primário, 2.924 tinham a informação sobre a necessidade ou não da repetição do ecocardiograma fetal. Em 2.788/3.263 (85,4\%) gestantes o ecocardiograma foi conclusivo e normal na primeira tentativa. Em 136/3.263 (4,2\%) houve a necessidade de repetílo na própria unidade de saúde, em visita posterior, para constatação da normalidade do ecocardiograma fetal, na maioria das vezes determinado pela precocidade da gestação ou devido à posição em que se encontrava o concepto no momento do primeiro exame. Na mesma população, foi possível visibilizar o coração pelo corte de quatro câmaras em $96,7 \%$ dos exames realizados. 0 septo interventricular foi identificado em $94,9 \%$ das vezes, a via de saída do ventrículo esquerdo em 94,2\% dos fetos e a via de saída do ventrículo direito em $90,4 \%$ dos exames (tab. III). Foi obtida imagem do arco aórtico em $71,6 \%$ das vezes.

Todos os 717 fetos examinados na Unidade de Cardiologia Fetal tiveram os cortes de quatro câmaras e vias de saída visibilizados, além de o equipamento utilizado permitir, em algumas oportunidades, diagnósticos auxiliados pelo uso do Doppler.

Os examinadores consideraram que $17 \%$ dos fetos da população de gestantes com origem nos postos de saúde tinham um motivo que dificultava a obtenção das imagens ecocardiográficas. Entre esses fatores, a posição em que se encontrava o concepto, a idade gestacional precoce e a obesidade materna foram os mais freqüentes. Em apenas 3,3\% das vezes o corte de quatro câmaras não foi obtido, havendo maior prejuízo sobre a obtenção da imagem do arco aórtico.

Foram encaminhados para ecocardiograma nas dependências da Unidade em nível terciário de atenção, por anormalidades suspeitas ou verificadas nos ecocardiogramas de triagem nos postos de saúde, 332/3.263 fetos, correspondendo a 10,2\% da população estudada, sendo que marcaram e realizaram o exame 176 gestantes (53\%).

Houve um total de 103 diagnósticos de anormalidades cardiovasculares fetais (tab. IV), correspondendo a uma prevalência global de 25,8/1000 (103/3.980), com um intervalo de confiança para $95 \%$ de 21,1 a 31,3. Destes, 81 (81/3.263 ou 2,4\%) foram

\begin{tabular}{|c|c|c|c|}
\hline \multicolumn{4}{|c|}{$\begin{array}{c}\text { Tabela II - Resultados dos ecocardiogramas de rastreamento, segundo } \\
\text { origem das gestantes }\end{array}$} \\
\hline & PS & UCF & TOTAL \\
\hline Normais & $3.182(80 \%)$ & $695(17,5 \%)$ & $3.877(97,5 \%)$ \\
\hline MCV & $37(0,9 \%)$ & $10(0,2 \%)$ & $47(1,1 \%)$ \\
\hline Golf Ball & $44(1,1 \%)$ & $12(0,3 \%)$ & $56(1,4 \%)$ \\
\hline Total & $3.263(82 \%)$ & $717(18 \%)$ & $3.980(100 \%)$ \\
\hline
\end{tabular}

\begin{tabular}{|lrrrrr|}
\hline \multicolumn{5}{|c|}{$\begin{array}{c}\text { Tabela III - Percentuais de visibilização dos cortes ecocardiográficos } \\
\text { fetais nas gestantes com origem nos postos de atendimento primário }\end{array}$} \\
\hline \multicolumn{7}{|c}{ 4C } & SIV & VE & VD & AA \\
\hline Normal & 95,3 & 93,5 & 93,9 & 90,0 & 71,2 \\
Alterado & 1,4 & 1,4 & 0,3 & 0,4 & 0,4 \\
Não visibilizado & 3,3 & 5,1 & 5,8 & 9,6 & 28,4 \\
\hline & 4C - quatro câmaras; SIV - septo interventricular; VE - via de saída do \\
ventrículo esquerdo; VD - via de saída do ventrículo direito; AA - arco aórtico.
\end{tabular}




\begin{tabular}{|c|c|c|c|c|c|}
\hline \multicolumn{6}{|c|}{$\begin{array}{l}\text { Tabela IV - Diagnósticos estabelecidos: frequiência absoluta (n) e } \\
\text { relativa (\%) segundo origem das gestantes, e prevalência específica } \\
\text { (por } 1000 \text { nascidos vivos) }\end{array}$} \\
\hline Diagnóstico & PS & UCF & Total & $\%$ & $\begin{array}{c}\text { Prevalência } \\
\text { específica }\end{array}$ \\
\hline Arritmias & 7 & 3 & 10 & 9,7 & 2,5 \\
\hline $\begin{array}{l}\text { Cardiopatia complexa } \\
\text { em xifópagos }\end{array}$ & 1 & . & 1 & 0,9 & 0,2 \\
\hline $\begin{array}{l}\text { Coarctação/interrupção } \\
\text { da aorta }\end{array}$ & 2 & - & 2 & 1,9 & 0,5 \\
\hline Comunicação interatrial & 2 & . & 2 & 1,9 & 0,5 \\
\hline Comunicação interventricular & 10 & 4 & 14 & 13,6 & 3,5 \\
\hline $\begin{array}{l}\text { Defeito septal atrioventricular } \\
\text { completo }\end{array}$ & . & 1 & 1 & 0,9 & 0,2 \\
\hline Derrame pericárdico & 3 & - & 3 & 2,9 & 0,7 \\
\hline Displasia tricúspide & 1 & . & 1 & 0,9 & 0,2 \\
\hline DVSVD + CIV subaórtica + EP & 1 & . & 1 & 0,9 & 0,2 \\
\hline Golfball & 44 & 12 & 56 & 54,3 & 14 \\
\hline $\begin{array}{l}\text { Hipoplasia de ventrículo } \\
\text { esquerdo }\end{array}$ & 2 & 1 & 3 & 2,9 & 0,7 \\
\hline Miocardiopatia hipertrófica & 6 & . & 6 & 5,8 & 1,5 \\
\hline Truncus arteriosus & 1 & . & 1 & 0,9 & 0,2 \\
\hline Tumor ventricular & 1 & . & 1 & 0,9 & 0,2 \\
\hline Veia cava superior esquerda & & 1 & 1 & 0,9 & 0,2 \\
\hline Total & 81 & 22 & 103 & 100 & 25,8 \\
\hline \multicolumn{6}{|c|}{$\begin{array}{l}\text { PS - postos de saúde; UCF - Unidade de Cardiologia Fetal; DVSVD - dupla } \\
\text { via de saída do ventrículo direito; CIV - comunicação interventricular; } \\
\text { EP - estenose pulmonar. }\end{array}$} \\
\hline
\end{tabular}

encontrados na população originária dos postos de saúde e 22 (22/717 ou $3,0 \%$ ) entre as gestantes que procuraram a Unidade para o exame de rastreamento. Os percentuais não são estatisti. camente diferentes entre si $\left(\chi^{2}=0,80 ; p=0,37\right)$.

Exibiam alterações de refringência (golf balls) 56 fetos (44 entre os examinados nos postos de saúde e 12 entre os realizados na Unidade).

As alterações morfo-funcionais fetais somaram 47 entre 3.980 exames, representando uma prevalência de malformações cardiovasculares de 11,8/1000 (intervalo de confiança 95\%: 8,6 a 15,6).

Tanto em um como em outro subgrupo as anormalidades mais freqüentes, excetuando as alterações de refringência, foram as comunicações interventriculares, seguidas pelas alterações de ritmo cardíaco (tab. IV).

Não houve nenhum falso-positivo entre os 3.980 fetos examinados e somente um teve morte intra-uterina. Nasceram vivos, portanto, 86 dos 87 fetos, com 5 óbitos pós-natais.

Na busca de falso-negativos, somente estavam disponíveis os dados de nascimentos referentes aos anos de 1998 e 1999, e parte de 2000 (dados parciais), representando 2.767 gestantes.

Foram localizados 828 nomes de mães de recém-nascidos no Sistema de Registro de Nascidos Vivos de Porto Alegre (SINASC) nos anos de 1998 a 2000. Os demais nomes também não constavam do registro da mortalidade do município, com exceção de 10, todos com atestado de óbito cuja causa básica excluía malformação cardiovascular.

Houve 3 falso-negativos (uma comunicação interventricular e coarctação aórtica, uma estenose pulmonar e aórtica e uma anomalia de Ebstein), elevando a prevalência global de malformações cardiovasculares fetais para 12,5/1000 (50/3.980, intervalo de confiança 95\%: 9,3 a 16,5), ou 26,6/1000 (106/3.980, intervalo de confiança 95\%: 21,8 a 32,1), caso incluídas também as alte316 rações de refringência.

\section{Discussão}

Os resultados obtidos no rastreamento das malformações cardiovasculares fetais na população estudada confirmam a experiência internacional ${ }^{24.33}$, com uma prevalência de malformações cardiovasculares no período pré-natal correspondente a 12,5/1000, onde foram identificadas pela ecocardiografia de rastreamento 47 alterações morfo-funcionais do coração fetal e 56 alterações na refringência endocárdica.

Embora ainda não suficientemente estudadas, e consideradas como alterações inespecíficas, golfballs podem se constituir em marcadores cardíacos de doenças sistêmicas e/ou cromossômicas, achados freqüentes nos cortes de quatro câmaras. Ocorrem em 3 a $8 \%$ das vezes, mais comumente nos músculos papilares do ventrículo esquerdo, mas também são visíveis no septo interventricular e no ventrículo direito. Histologicamente, representam um excesso do depósito de cálcio no miocárdio ${ }^{34}$.

Entre as alterações morfo-funcionais, a comunicação interventricular, diagnosticada em 14 fetos, foi a doença mais freqüentemente encontrada, concordando com a literatura internacional.

Hafner e cols. ${ }^{32}$, em estudo com delineamento muito similar ao nosso, relataram que os defeitos do septo ventricular são os mais comumente identificados, embora apenas $10 \mathrm{em} 36$ tenham tido diagnóstico pré-natal. Relataram também que a prevalência encontrada (13,3/1000), mais elevada que a esperada (em torno de $8 / 1000$ ), provavelmente se devesse à procura mais insistente por malformações cardiovasculares que a produzida por outros autores.

As arritmias constituem-se em outro grupo de doenças muito freqüentes no período intra-uterino, sendo causa potencial de hidropisia, morte súbita e óbito fetal, quando não reconhecidas e adequadamente acompanhadas.

O corte de quatro câmaras é o mais importante na triagem para malformações cardiovasculares e esteve alterado em $60 \%$ das malformações maiores ${ }^{35}$.

Embora a maioria das anormalidades que afetam os lados direito ou esquerdo do coração determinem uma imagem de quatro câmaras anormal, os mais comuns defeitos dos grandes vasos, como tetralogia de Fallot e transposição dos grandes vasos, freqüentemente são associados com uma imagem de quatro câmaras normal na ecocardiografia fetal.

Vários autores propuseram a adição das imagens das vias de saída direita e esquerda à imagem de quatro câmaras para incrementar os índices de deteç̧ão pré-natal de defeitos cardiovascu$\operatorname{lares}^{24,26,27,36 \cdot 39}$.

DeVore relata que a visibilização das vias de saída, embora requeira algum treinamento adicional, é possível em $97 \%$ dos ecocardiogramas fetais. Nesse estudo, em $90 \%$ das vezes, foram visibilizadas a via de saída do ventrículo direito e em 93,9\% a via de saída do ventrículo esquerdo ${ }^{36}$.

O emprego sistemático da visibilização dos cortes das vias de saída do ventrículos direito e esquerdo permitiu a visibilização adicional de 4 (8,9\%) cardiopatias (duas coarctação/interrupção do arco aórtico, uma dupla via de saída do ventrículo direito e um truncus arteriosus), teoricamente não detectáveis ao corte de quatro câmaras isoladamente.

A obtenção de outros cortes como arcos ductal e aórtico não contribui para a incrementar o diagnóstico pré-natal ${ }^{40}$. 
Bromley e cols., estudando a acurácia dos cortes de quatro câmaras e vias de saída dos ventrículos direito e esquerdo na detecção de cardiopatias ainda no período pré-natal, relataram que, em mãos experientes, esses cortes são obtidos em mais de 95\% das vezes em fetos de 18 ou mais semanas, sendo possível detectar $83 \%$ das malformações cardiovasculares ${ }^{37}$.

Vários estudos relatam que o corte de quatro câmaras é ca. paz de detectar de 5 a $81 \%$ dos defeitos cardíacos fetais ${ }^{28,41 \cdot 45}$. 0 acréscimo das vias de saída direita e esquerda em um ecocardiograma pode elevar o índice para 14 a 83\% 29,37.

Na nossa população, em apenas 3,3\% das vezes não foi possível visibilizar o corte de quatro câmaras, em 5,8\% a via de saída do ventrículo esquerdo e em $9,6 \%$ a via de saída do ventrículo direito, devido, principalmente, à posição em que se encontrava o feto, à idade gestacional precoce ou ao excesso de peso materno.

A inclusão do mapeamento em cores pelo Doppler colorido não é essencial para o reconhecimento anatômico de corações anormais, sendo doenças identificáveis com o exame bidimensional isolado ${ }^{46}$. Como esta tecnologia agrega custos significativos na aquisição de equipamentos, a constatação reduz o custo da triagem pré-natal, já que permite o emprego de ecógrafos não tão sofisticados.

Foi detectada a ocorrência de 3 recém-nascidos portadores de malformação cardiovascular, considerados normais no exame pré-natal (falso-negativos). Um, detectado através da metodologia de busca das informações pós-natais junto aos serviços de cardiologia pediátrica e maternidades da cidade, portador de comunicação interventricular mínima e coarctação da aorta. Os outros 2 tiveram exames de triagem considerados normais pela equipe que visitou o posto de atenção primária, mas foram referidos à Unida. de de Cardiologia Fetal em semanas subseqüentes por alteração à ultra-sonografia obstétrica, ainda a tempo de diagnosticar as doenças que eram portadores (um com anomalia de Ebstein e outro com estenose severa das artérias pulmonar e aorta) antes do nascimento do concepto. Na realidade, o diagnóstico ainda no período intra-uterino foi estabelecido, mas em nome do rigor científico devem ser considerados como falso-negativos, visto terem sido rastreados e liberados como normais. A explicação mais provável parece residir na precocidade da ecocardiografia de triagem (exames realizados com 18 e 20 semanas).

Essas anomalias, especialmente os pequenos defeitos septais atriais e ventriculares, freqüentemente não são visibilizados nos ecocardiogramas de rastreamento ${ }^{47}$. Segundo Leslie e cols., esses defeitos, juntamente com a coarctação aórtica, são os responsá. veis pelo maior número de falso-negativos ${ }^{48}$.

Dos falso-negativos, o primeiro corresponde a uma doença com reconhecida dificuldade de visibilização aos cortes de triagem e com o equipamento básico empregado. Benacerraf e cols. diag. nosticaram apenas 4 fetos com coarctação aórtica entre 9 que tinham como suspeita um aparente maior tamanho do ventrículo direito em relação ao esquerdo ao corte de quatro câmaras ${ }^{49}$. Hornberger e cols. ressaltaram que o diagnóstico intra-uterino de coarctação aórtica é ainda um desafio, e, em estudo multicêntrico delineado para identificar características ecocardiográficas fetais, que auxiliassem a sua observação, relataram que a hipoplasia quantitativa do arco distal (arco transverso e istmo) é o achado com maior valor na predição de coarctação ${ }^{50}$.
Vários relatos na literatura fazem referência ao controle clínico pós-natal, incluídos aqueles com pretensão de estabelecer prevalência de cardiopatias congênitas no período pré-natal ${ }^{51}$. Os estudos iniciais de avaliação do papel de corte de quatro câmaras no rastreamento de malformações cardiovasculares fetais, em centros de referência e com tamanho de amostra não muito expressivo, fizeram do controle clínico pós-natal, referido pelo pediatra no acompanhamento do lactente, o método de obtenção da informação sobre os falso-negativos ${ }^{32,33,38,40,51}$.

Deve-se reconhecer a possibilidade de existirem outros fetos portadores de malformações cardiovasculares que não tenham sido diagnosticadas pela ecocardiografia de rastreamento, já que não foi utilizado o padrão ouro para o controle pós-natal, absolutamente inexequíivel no estágio atual de organização da assistência em cardiologia pediátrica em nosso meio. Entretanto, este fato vem apenas reforçar a observação de que a prevalência de malformações cardiovasculares deva ser maior no período pré-natal.

Embora não tenha sido nosso objetivo determinar a sensibilidade (dependente do verdadeiro número de malformações cardiovasculares aferida por padrão ouro metodológico) e a especificidade (dependente dos falso-positivos), podemos calculá-las a partir dos resultados encontrados. Como foram encontrados 3 falso-negativos (elevando o número total de malformações cardiovasculares da população estudada para 50), a sensibilidade ficou, então, estabelecida em 94\% (47/50), e a especificidade em 100\%, já que não houve falso-positivos.

O valor preditivo positivo, aferição dos verdadeiro-positivos entre os que têm o teste positivo, também é dependente da detecção de falso-positivos. Como nenhum feto, diagnosticado como portador de malformação cardiovascular, foi no período pós-natal constatado como normal, o valor preditivo positivo do teste, na população em estudo, é de $100 \%$.

Pela constatação dos 3 falso-negativos, o valor preditivo negativo, aferição dos verdadeiro-negativos entre os que tiveram triagem considerada normal, foi 99,9\% (3.874/3.877).

Apesar de fragilizado pela decisão metodológica de não realização de ecocardiograma em cores com Doppler no período pósnatal para controle em todos os fetos (padrão ouro reconhecido), e pela não realização de necropsia sistemática em todos os óbitos (fetais e neonatais), há um razoável grau de convencimento no sentido de que a prevalência aqui encontrada represente um índice muito próximo do verdadeiro no município de Porto Alegre.

Mesmo quando os resultados e eficácia da triagem pré-natal das malformações cardiovasculares são colocados em dúvida, deve ser levado em conta que o planejamento perinatal inegavelmente aumenta as chances de sobrevida do feto malformado.

Embora praticamente todos os defeitos estruturais cardíacos detectáveis pelo corte de quatro câmaras possam resultar em óbito nos primeiros anos de vida da criança, sua mortalidade pode ser reduzida pelo diagnóstico pré-natal ${ }^{52}$.

A mortalidade infantil verificada no Rio Grande do Sul e, mais especificamente, em Porto Alegre, onde as malformações cardiovasculares são responsáveis por $48 \%$ dos óbitos por anomalias congênitas e por 7,9\% de todos os óbitos em menores de um ano, é muito semelhante à mostrada em trabalho realizado na região norte inglesa ${ }^{2}$. Entre 230.654 nascimentos, $43 \%$ de todos os óbitos por malformações congênitas e $9 \%$ de toda a mortalidade infantil foram por malformações cardíacas. Ainda nesse trabalho,

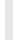

.


Abu-Harb e cols. chamam a atenção para o fato de que somente $70 \%$ das cardiopatias haviam sido diagnosticados antes do óbito.

Dentre as 47 alterações morfo-funcionais encontradas, pelo menos $10(21,2 \%)$ exigiam planejamento da atitude a ser toma. da no período neonatal imediato, pelo potencial de repercussões hemodinâmicas que poderiam desencadear.

Vale lembrar que o sistema de atendimento perinatal em Porto Alegre, em especial o neonatal, está sobrecarregado de pacientes, da cidade e região metropolitana, e das cidades do interior do Estado, com lotação de suas Unidades de Terapia Intensiva Neo. natais sempre saturada.

Recém-nascidos sem diagnóstico pré-natal contribuem, e muito, para aumentar o caos do setor, já que a maioria das Unida. des não dispõe de profissionais e equipamentos para o diagnóstico diferencial com cardiopatias congênitas no âmbito da própria UTI, carecendo de transporte intra ou inter-hospitalar, agregando custos e riscos aos recém-nascidos, sem contar o tempo, muitas vezes literalmente vital, que é dispendido até a elucidação diagnóstica.

Oliveira e cols..$^{53}$ já tiveram a oportunidade de relatar que $74 \%$ (59/80) das mães de recém-nascidos com cardiopatia congênita internados na UTI Pediátrica do Instituto de Cardiologia haviam realizado pelo menos um exame ecográfico durante a gestação, mas 54 deles $(91,6 \%)$ não tiveram a suspeita levantada pela ultrasonografia obstétrica, sugerindo que o coração fetal não estivesse sendo avaliado de maneira sistemática pelos seus realizadores.

DeVore, estimando os custos para a detecção de uma cardiopatia nos Estados Unidos em unidades sem treinamento específico para o diagnóstico pré-natal, afirmou ser muito mais produtiva a criação de uma rede sob a supervisão de especialistas para incrementar o número de diagnósticos pré-natais de malformações cardiovasculares ${ }^{54}$.

Somos um povo que, culturalmente, não aceita a necropsia com facilidade e poucos são os casos diagnosticados ou confirma. dos por ela. A alternativa exeqüível para determinar a prevalência de malformações cardiovasculares em nosso meio é a ecocardiografia, e o momento de realizá-la é na gestação.

Aerts e cols ${ }^{55}$, categorizando os óbitos infantis de 1997 a 1999 em Porto Alegre, de acordo com a causa básica e classificando-os de acordo com o seu potencial de redutibilidade, ressaltam a im. pressionante percentagem de 94,1\% (274/291) de óbitos de menores de um ano em 1999 considerados como evitáveis e a inexpressividade das doenças imunopreveníveis na mortalidade infantil (refletindo o intenso investimento realizado até agora na área). Outro item constitui-se na significativa participação do componente prénatal no potencial de redutibilidade (responsável por 49,4\% das mortes neonatais evitáveis, ou 84/164 bebês), mostrando que muito ainda (e já) deve ser feito para se obter um controle mais adequado das gestações visando a diminuição da mortalidade infantil.

Mas o destaque deste trabalho é o maior número de óbitos 141/372(37,9\%) em 1997, 174/377 (46,1\%) em 1998, e $127 /$ 291 (43,6\%) em 1999 no grupo dos redutíveis por diagnóstico e tratamento precoces, representando cerca de um terço dos óbitos neonatais e mais da metade da mortalidade pós-natal. Sendo as cardiopatias congênitas o principal grupo de causas de mortalidade por anomalias congênitas, com representação tão expressiva na mortalidade infantil em números absolutos e relativos, res. ponsável por significativa morbidade, nada mais lógico que um 318 dos passos seguintes no planejamento da estratégia para enfren- tar esse quadro e diminuir a mortalidade seja o da articulação dos setores já organizados e com vocação para o desempenho dessa tarefa, aliado à decisão política do poder público de investimentos em recursos humanos e tecnológicos.

Pelos dados levantados, há razões justificadas para considerar o município de Porto Alegre (e, talvez, também o Estado do Rio Grande do Sul) maduro para implementar um programa de rastreamento de malformações cardiovasculares fetais.

Como proposta concreta, vislumbramos um pré-natal em que fossem ofertados no mínimo dois ecogramas obstétricos a cada gestante: um, precoce, destinado a determinar confiavelmente a idade gestacional e, outro, após 20-24 semanas, a fim de estudar a morfologia do concepto e acompanhar a gestação. Neste 20 exame, seria incluída a visibilização rotineira do coração fetal, entre outros focos de interesse, mediante a realização dos cortes de quatro câmaras e das vias de saída direita e esquerda. A realização da triagem rotineira pré-natal para malformações cardiovasculares estaria, então, viabilizada em escala. Os ecografistas participantes do sistema a ser criado, já qualificados para a prática profissional gineco-obstétrica, receberiam treinamento específico adicional na obtenção e interpretação de imagens do coração fetal, sob supervisão sistemática e continuada da Unidade de Cardiologia Fetal do Instituto de Cardiologia do Rio Grande do Sul.

A visibilização de uma imagem suspeita, ou a mera dúvida quanto à sua presença, remeteria a gestante e seu feto para ecocardiograma fetal em nível secundário de atenção. Aos casos confirmados seria assegurada a continuidade de acompanhamento pela equipe multidisciplinar da Unidade de Cardiologia Fetal, utilizando-se recursos disponíveis para terapêutica ainda intra-útero, ou fazendo-se o planejamento do atendimento perinatal.

Imaginamos, assim, dotar a cidade de um instrumento capaz de assistir a população de maneira eficiente, gerando conhecimento científico, permitindo sua ampliação e multiplicando de maneira modular a experiência, expandindo-se para a região metropolitana de Porto Alegre e daí para todo o Estado. Ressalte-se aqui que a proposta poderia ser implementada de maneira progressiva, em conformidade com os recursos disponíveis em cada localidade, mas tendo como objetivo final a implantação efetiva de uma rede de triagem para malformações cardiovasculares fetais.

Em conclusão, a prevalência global de anormalidades morfofuncionais do sistema cardiovascular fetal, detectável pelos cortes ecocardiográficos de quatro câmaras e vias de saída dos ventrículos direito e esquerdo, em população de gestantes de baixo risco no município de Porto Alegre, é de 12,5/1000. A prevalência específica de 3,5/1000 para comunicação interventricular, de 2,5/ 1000 para distúrbios do ritmo cardíaco fetal, 1,5/1000 para miocardiopatia hipertrófica fetal, de 0,7/1000 para hipoplasia do ventrículo esquerdo e derrame pericárdico, de 0,5/1000 para coarctação/interrupção do arco aórtico e comunicação interatrial, e de 0,2/1000 para cardiopatia complexa em xifópagos, displasia tricúspide, dupla via de saída do ventrículo direito com comunicação interventricular subaórtica, truncus arteriosus, tumor ventricular e veia cava superior esquerda.

Ainda temos muito a fazer em termos de saúde coletiva, mas é inegável que cerca de $50 \%$ da mortalidade infantil prevenível ou evitável em Porto Alegre esteja na dependência de investimentos em tecnologias e recursos humanos capazes de fazer frente a este desafio. 


\section{Referências}

1. Hoffman JIE. Incidence of congenital heart disease: I. Postnatal incidence. Pediatr Cardiol 1995; 16: 103.13.

2. Abu-Harb M, Hey E, Wren C. Death in infancy from unrecognised congenital heart disease. Arch Dis Child 1994; 71: 3.7.

3. PráSaber-Informações de Interesseà Saúde-Sistema de Informação sobre Mor talidade. SIM 1997/1998. Prefeitura Municipal de Porto Alegre, Secretaria Municipal de Saúde, Coordenadoria Geral de Vigilância em Saúde, Equipe de Informações em Saúde 1999; 4: 10-149.

4. PráSaber-Informações de Interesseà Saúde-Porto Alegreem números: dados populacionais, nascidos vivos, mortalidade 1998. Prefeitura Municipal de Porto Ale gre, Secretaria Municipal de Saúde, Coordenadoria Geral de Vigilância em Saúde, Equipe de Informações em Saúde 1999;4: 13-105.

5. Estatísticas de Saúde- Mortalidade. Publicação da Secretaria Estadual da Saúde e Meio Ambiente do Rio Grande do Sul, 1992; 18: 1-160.

6. Fixler DE. Trends in congenital heartdiseases in Dallas County births. Circulation 1990; 81: 137.42.

7. Wren C, Richmond S, Donaldson L. Temporal variability in birth prevalence of car diovascular malformations. Heart 2000; 83: 414.9.

8. Borman B, Chapman C, Howard K, BuckfieldP, Findlay J. Using a national register for the epidemiological study of congenital heart defects. NZ Med J 1987; 100 : 404.6.

9. Carlgren LE, Ericson A, Källén B. Monitoring of congenital cardiac defects. Pediatr Cardiol 1987; 8: 247.56.

10. Centers for Disease Control. Congenital Malformations Surveillance Jan 1982-Dec 1985. US Dept Health Human Services, PHS, Atlanta, 1988.

11. FerenczC, Villaseñor AC. Epidemiology of cardiovascular malformations: The state of the art. Cardiol Young 1991; 1:264-84.

12. Gabritz RG, Joffre MR, Collins-Nakai RL. Congenital heart disease: Incidence in the first year of life. Am J Epidemiol 1988; 128: 81-88.

13. Samánek M, SlavikZ, Zborilová B, Hrobonová V, Vorískova M, Skovránek J. Pre. valence, treatment, and outcome of heart disease in live-born children: a prospective analysis of 91,823 live-born children. Pediatr Cardiol 1989; 10:205-11.

14. Samánek M, Voriskova M. Congenital heart disease among 815,569 children born between 1980 and 1990 and their 15 -year survival: a prospective Bohemia survival study. Pediatr Cardiol 1999;20:411.7.

15. Stoll C, Alembik Y, Roth MP, Dott B, De Geeter B. Risk factors in congenital heart disease. Eur J Epidemiol 1989; 5: 382-91.

16. Bound JP, Logan WFWE. Incidence of congenital heart disease in blackpool. $\mathrm{Br}$ Heart J 1977; 39: 445-50.

17. Hoffman JIE, Christianson R. Congenital heart disease in a cohort of 19,502 births with long.term follow up. Am J Cardiol 1978; 42:641-7.

18. Hagemann LL. Estudo pré-natal da miocardiopatia hipertrófica e sua associação com os níveis de insulina em fetos de mães diabéticas [Dissertação de Mestrado]. Porto Alegre: Departamento de Pediatria-UFRGS, 1994.

19. Mikamo K. Anatomic and chromosomal studies in spontaneous abortions. Am J Obstet Gynecol 1970; 106: 243.54.

20. Gerlis LM. Cardiac malformations in spontaneous abortions. Int J Cardiol 1985; 7:29-43.

21. Poland BJ, Lowry RB. The use of spontaneous abortions and stillbirths in genetic couselling. Am J Obstet Gynecol 1974; 118: 322-6.

22. Hoffman JIE. Incidence, mortality and natural history. In: Anderson RH, Macartney FJ, Shinebourne EA, et al. (eds). Pediatric Cardiology. London: Churchil Li. vingstone, 1987: 3-14.

23. Allan LD. Echocardiographic detection of congenital heart disease in the fetus: present and future. Br Heart J 1995; 74: 103.6.

24. Wigton TR, Sabbagha RE, Tamura RK, Cohen L, Minogue JP, Strasburger JF. So nographic diagnosis of congenital heart disease: comparison between the fourchamber view and multiple cardiac views. Obstet Gynecol 1993; 82:219-24.

25. Stumpflen I, Stumpflen A, Wimmer M, Bernaschek G. Effect of detailed fetal echocardiography as part of routine prenatal ultrasonographic screening on detection of congenital heart disease. Lancet 1996; 348(9031): 854-7.

26. Achiron R, Glaser J, Gelernter I, Hegesh J, Yagel S. Extended fetal echocardiogra phic examination for detecting cardiac malformations in low risk pregnancies. $\mathrm{Br}$ Med J 1992; 304(6828): 671-4.

27. Stoll C, Alembik Y, DottB, Roth PM, De Geeter B. Evaluation of prenatal diagnosis of congenital heart disease. Prenat Diagn 1993; 13: 453-61.

28. Vergani P, Mariani S, Ghidini A, Schiavina R, Cavallone M, Locademi A, et al . Screening for congenital heart disease with the four-chamber view of the fetal heart. Am J Obstet Gynecol 1992; 167: 1000-3.
29. KirkJS, ComstockCH, LeeW, Smith RS, Riggs TW, WeinhouseE. Sonographic screening to detect fetal cardiac anomalies: a 5-year experience with 111 abnormal cases. Obstet Gynecol 1997; 89:227-32

30. Todros T, Faggiano F, Chiappa E, Gaglioti P, Mitola B, Sciarrone A. Accuracy of routine ultrasonography in screening heart disease prenatally. Gruppo Piemontese for Prenatal Screening of Congenital Heart Disease. Prenat Diagn 1997; 17:901.6.

31. OttWJ. The accuracy of antenatal fetal echocardiography screening in high- and low-risk patients. Am J Obstet Gynecol 1995; 172: 1741-7.

32. Hafner E, Scholler J, Schuchter K, SternisteW, Philipp K. Detection of fetal congenital heart disease in a low-risk population. Prenat Diagn 1998; 18: 808-15.

33. Buskens E, Grobbee DE, Frohn-Mulder IME, Stewart PA, Juttman RE, Wladdimiroff JW, et al. Efficacy of routine fetal ultrasound screening for congenital heart disease in normal pregnancy. Circulation 1996; 94:67-72.

34. Allan LD. Indications for fetal echocardiography. In: Allan LD, Hornberger L, Sharland G(eds). Textbook of Fetal Cardiology. Greenwich Medical Media, 2000:47-53.

35. Allan LD. Manual of fetal echocardiography. Lancaster, MTP Press Limited, 1986.

36. DeVore GR. Fetal cardiac outflow tract screening J Ultrasound Med 1992; 12: 645.646.

37. Bromley B, Estroff JA, Sanders SP, Parad R, Roberts D, Frigoletto FD Jr., Benacerraf BR. Fetal echocardiography: accuracy and limitations in a population at high and low risk for heart defects. Am J Obstet Gynecol 1992; 166: 1473-81.

38. Bronshtein M, ZimmerEZ, Gerlis LM, Lorber A, Drugan A. Early ultrasound diag. nosis offetal congenital heart defects in high-risk and low-risk pregnancies. Obstet Gynecol 1993; 82: 225.9.

39. Benacerraf BR. Sonographic detection of fetal anomalies of the aortic and pulmonary arteries: value of four-chamber view vs direct images. Am J Roentgenol 1994; 163: 1483-9.

40. Smith RS, ComstockCH, Lorenz RP, Kirk JS, LeeW. Maternal diabetes mellitus: which views are essential for fetal echocardiography? Obstet Gynecol 1997; 90 : 575-9.

41. ConstantineG, McCormack J. Comparative audit of booking and mid-trimesterultrasound scans in the prenatal diagnosis of congenital anomalies. Prenat Diagn 1991; 11:905-14.

42. LuckCA. Value of routine ultrasound scanning at 19 weeks: a four year study of 8849 deliveries. Br Med J 1992; 304: 1474-8.

43. KirkJS, Riggs TW, ComstockCH, LeeW, YangSS, Weinhouse E. Prenatal screening for cardiac anomalies: the value of routine addition of the aortic root to the four. chamberview. Obstet Gynecol 1994;84:427.31.

44. Tegnander E, Eik-Nes SH, Johansen OJ, Linker DT. Prenatal detection of heartdefects at the routine fetal examination at 18 weeks in a non-selected population. UI. trasound Obstet Gynecol 1995; 5: 372-80.

45. KirkJS, ComstockCH, LeeW, Smith RS, Riggs TW, WeinhouseE. Fetal cardiac asymmetry: a markerfor congenital heart disease. Obstet Gynecol 1999; 93: 189-92.

46. Copel JA, Morotti R, Hobbins JC, Kleinman CS. The antenatal diagnosis of congenital heart disease using fetal echocardiography: is color flow mapping necessary? Obstet Gynecol 1991; 78: 1-8.

47. Mcgahan J. Sonography of the fetal heart: findings on the four-chamberview. Am J Roentgenol 1991; 156: 547.53

48. Leslie KK, PersutteWH, Drose JA, Lenke RR, Hobbins JC, Manco-Johnson M, etal. Prenatal detection of congenital heart disease by basic ultrasonographyatatertiary care center: what should our expectations be? J Matern Fetal Invest 1996; 6: 132-5.

49. Benacerraf BR, SaltzmanDH, Sanders SP.Sonographic sign suggesting the prenatal diagnosis of coarctation of the aorta. JUltrasound Med 1989; 8: 65-9.

50. Hornberger LK, Sahn DJ, Kleinman CS, Copel JA, Silverman, NH. Antenatal diagnosis of coarctation of the aorta: a multicenter experience. J Am Coll Cardiol 1994; 23:417.23.

51. Copel JA, Pila G. Fetal echocardiographic screening for congenital heart disease: the importance of four chamberview. Am J Obstet Gynecol 1987; 157: 648-55.

52. Saari-Kemppainen A, Karjalainen O, Ylostalo P, Heinonen OP. Ultrasound screening and perinatal mortality: controled trial of systematic one stage screening in pregnancy. Lancet 1990; 336: 387.91.

53. Oliveira LT, BonowFP, Ceccon G, Zimmer LP, Hagemann LL, Zielinsky P.O papel da ultra-sonografia obstétrica de rotina no diagnóstico presuntivo de cardiopatias. RevAMRIGS 1997; 41: 181-6.

54. DeVore GR. Influence of prenatal diagnosis on congenital heart defects. Ann NY Acad Sci 1998; 847: 46-52.

55. Aerts D, Cunha J, Hilgert C, Sant'Anna A. A mortalidade infantil em Porto Alegre: até quanto podemos reduzir? Anais do Congresso Brasileiro de Saúde Coletiva, Salvador, Bahia, 08/2000. 